Pacific Journal of Mathematics

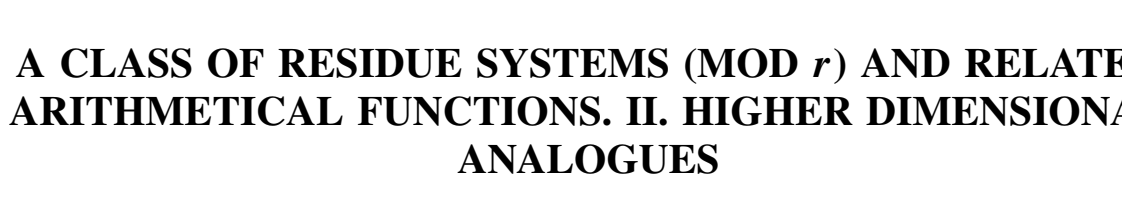




\section{A CLASS OF RESIDUE SYSTEMS (mod $r$ ) AND RELATED ARITHMETICAL FUNCTIONS, II. HIGHER DIMENSIONAL ANALOGUES}

\section{ECKFORD COHEN}

1. Introduction. In an earlier paper [3] with a similar name (to be referred to as I) we introduced the idea of a direct factor set $(P$-set) and the residue system $(\bmod n)$ associated with such a set. We first review briefly these concepts. Two non-vacuous subsets $P, Q$ of the positive integers $Z$ are said to form a conjugate pair of direct factor sets provided the following two conditions are satisfied:

(i) an integer $n>0$ is in $P$ (or $Q$ ) if and only if, for each factorization, $n=n_{1} n_{2},\left(n_{1}, n_{2}\right)=1, n_{1}$ and $n_{2}$ are also in $P$ (or $Q$ ),

(ii) every positive integer $n$ possesses a unique factorization of the form, $n=a b$ such that $a \in P, b \in Q$. A set of integers $a(\bmod n)$ such that $(a, n) \in P$ is said to form a $P$-reduced residue system $(\bmod n)$, or $P$-system $(\bmod n)$, and the number of elements in such a system is denoted by $\phi_{P}(n)$. The fundamental result of I was a generalization of the Möbius inversion formula to conjugate pairs of direct factor sets. This result is reformulated in $\S 2$ of the present paper.

In this paper we extend the notion of a $P$-system $(\bmod n)$ from the set of integers $X$ to $t$-dimensional vectors over $X$ (briefly, $X_{t}$-vectors), $t \geqq 1$. The one dimensional case $(t=1)$ is the case already investigated in I. Two $X_{t}$-vectors, $A=\left\{a_{i}\right\}, B=\left\{b_{i}\right\}$, are said to be congruent $(\bmod t, n)$, written $A \equiv B(\bmod t, n)$, provided $a_{i} \equiv b_{i}(\bmod n), i=1, \cdots, t$. Moreover, we place $\left(a_{i}\right)=\left(a_{i}, \cdots, a_{t}\right)$, using the convention, $(0, \cdots, 0)=0$, and define vector sums and scalar multiples in the usual way. A $P$ reduced residue system $(\bmod t, n)$, or $P$-system $(\bmod t, n)$, is defined to be a maximal set of mutually incongruent $X_{t}$-vectors $(\bmod t, n),\left\{a_{i}\right\}$, satisfying $\left(\left(a_{i}\right), n\right) \in P$. The number of elements in such a system depends only on $t$ and $n$, and is denoted $J_{t, P}(n)$ and called the $(t, P)$-totient of $n$. In case $P$ is the unit set $1, J_{t, P}(n)$ reduces to the ordinary Jordan totient, $J_{t, 1}(n)=J_{t}(n) . \quad$ A $P$-system with $P=Z$ is called a complete residue system $(\bmod t, n)$; clearly $J_{t, z}(n)=n^{t}$.

REMARK 1.1. An $X_{t}$-vector whose components are in $Z$ will be called a $Z_{t}$-vector, and a $P$-system $(\bmod t, n)$ consisting of elements of $Z_{t}$ alone will be called a positive $P$-system $(\bmod t, n)$.

We summarize now the salient points of the paper. In $\S 2$ an enumerative principle for $X_{t}$-vectors (Theorem 2.1) is formulated, generalizing a result proved in $[3, \S 3]$ in the case $t=1$. This result is used,

Received January 30, 1959. 
in conjunction with the inversion principle of $I$, to obtain an evaluation of $J_{t, P}(n)$. A function $\phi_{\alpha, P}(n)$, formally generalizing $J_{t}(n)$, is also introduced, along with a generalized divisor function $\sigma_{\alpha, p}(n)$. Certain closely related functions, $\phi_{\alpha, P}^{*}(n)$ and $\sigma_{\alpha, P}^{*}(n)$ are also defined in $\S 2$.

In $\S 3$ we introduce the zeta function $\zeta_{P}(s)$ associated with a direct factor set $P$. In case $P=Z, \zeta_{P}(s)$ is the ordinary $\zeta$-function, $\zeta(s)$. Employing the generalized inversion function $\mu_{P}(n)$ of $\mathrm{I}$ we also define "reciprocal" $\zeta$-functions $\tilde{\zeta}_{P}(s)$ and obtain in (3.8) a generalization $(P=1$, $Q=Z$ ) of the familiar fact,

$$
\sum_{n=1}^{\infty} \frac{\mu(n)}{n^{s}}=\zeta^{-1}(s)
$$

where $\mu(n)$ denotes the Möbius function. Broad generalizations of other basic identities involving $\zeta$-functions are also deduced.

In $\S 4$ we obtain mean value estimates for the functions $\phi_{\alpha, P}(n)$ and $\sigma_{\alpha, P}(n)$, valid for arbitrary direct factor sets $P$, extending basic properties of $\phi(n)$ and $\sigma(n)=\sigma_{1, z}(n)$. For example, (4.5) reduces in case $\alpha=1$, $P=1$, to the celebrated result [1, Theorem 330] of Mertens for the Euler $\phi$-function,

$$
\sum_{n \leqq x} \phi(n)=\frac{3 x^{2}}{\pi^{2}}+O(x \log x) .
$$

Using results of $\S 4$, we obtain in $\S 5$ (Theorem 5.1 ) for $t \geqq 2$, the asymptotic density of $Z_{t}$-vectors $\left\{a_{i}\right\}$, such that $\left(a_{i}\right) \in P$. Numerous special cases are considered (Corollary 5.2). We mention that Corollary 5.3, in case $t=2$, yields a result of Kronecker asserting that the density of the integral pairs with a fixed greatest common divisor $r$ is $6 / \pi^{2} r^{2}$.

In $\S 6$ we generalize the so-called "second Möbius inversion formula" to conjugate sets $P, Q$ (Theorem 6.1). Application of this extended inversion relation yields in (6.3) a generalization of broad scope of Meissel's well known identity,

$$
\sum_{1 \leqq n \leqq x} \mu(n)\left[\frac{x}{n}\right]=1 .
$$

We also evaluate in $\S 6$ a generalization to $P$-sets of Legendre's totient function $\phi(x, n)$, defined to be the number of integers a such that $1 \leqq a \leqq x,(\mathrm{a}, n)=1$.

REMARK 1.2. It is noted that many of the results of this paper are valid, not merely for direct factor sets, but for quite arbitrary sets of integers $P$. For example, this is true in the case of Corollary 5.1. Moreover, a number of the remaining results can be reformulated in such a manner as to be valid for arbitrary sets $P$. We shall restrict our attention, however, to direct factor sets, reserving the treatment of more 
general sets for a later paper, to be based on other methods. The advantage of a separate treatment of direct factor sets arises from the applicability of the generalized inversion theorem.

2. Generalized totient and divisor functions. Let $P$ and $Q$ denote an arbitrary conjugate pair of direct factor sets, and define, as in I,

$$
\begin{aligned}
& \rho_{P}(n)= \begin{cases}1 & (n \in P) \\
0 & (n \notin P),\end{cases} \\
& \mu_{P}(n)=\sum_{\alpha=n} \rho_{P}(d) \ell_{\ell}(\delta) .
\end{aligned}
$$

The functions $\rho_{P}(n)$ and $\mu_{P}(n)$ are termed, respectively the characteristic function and inversion function of the set $P$. The inversion formula of I can be restated in the form,

$$
f(n)=\sum_{d \delta=n} \rho_{Q}(d) g(\delta) \rightleftarrows g(n)=\sum_{d \delta=n} \mu_{P}(d) f(\delta) .
$$

This principle is a direct consequence of the relation,

$$
\sum_{a \delta=n} \mu_{P}(d) \rho_{Q}(\delta)=\rho(n),
$$

where $\rho(n)=\rho_{1}(n)$ (that is, $\rho(n)=1$ or 0 according as $n=1$ or $n>1$ ). Note that $\mu_{P}(n)$ reduces to $\mu(n)$ when $P=1$.

In order to evaluate $J_{t, P}(n)$, we shall need the following results generalizing Theorem 4 of I to $t$ dimensional vectors.

THEOREM 2.1. If $d$ ranges over the divisors of $n$ contained in $Q$, and for each $d, x$ ranges over the elements of a P-system $(\bmod t, \delta)$, $d \delta=n$, then the set $d x$ constitutes a complete residue system $(\bmod t, n)$.

We omit the proof, which is analogous to the proof in case $t=1$. On the basis of this result it follows immediately that

$$
\sum_{l \delta=n} \rho_{Q}(d) J_{t, P}(\delta)=n^{t} .
$$

Application of (2.3) to (2.5) yields

\section{THEOREM 2.2.}

$$
J_{t, P}(n)=\sum_{d \delta=n} d^{t} \mu_{P}(\delta)
$$

Define now for $\alpha$ an arbitrary real number, the generalized totient,

$$
\phi_{\alpha, P}(n)=\sum_{a \delta=n} d^{\alpha} \mu_{P}(\delta),
$$

so that $\phi_{\alpha, P}=J_{t, P}(n)$ in case $\alpha=t$ is a positive integer. We also define analogously a generalized divisor function by placing 


$$
\sigma_{\alpha, P}(n)=\sum_{a \delta=n} d^{\alpha} \rho_{P}(\delta)=\sum_{\substack{a \delta=n \\ \delta \in P}} d^{\alpha} .
$$

Corresponding to the functions $\phi_{\alpha, P}(n), \sigma_{\alpha, P}(n)$ we define related functions,

$$
\begin{aligned}
& \phi_{a, P}^{*}(n)=\sum_{a \mid n} d^{\alpha} \mu_{P}(d) \\
& \sigma_{\alpha, P}^{*}(n)=\sum_{a \mid n} d^{\alpha} \rho_{P}(d)=\sum_{\substack{a \mid n \\
a \in P}} d^{\alpha} .
\end{aligned}
$$

The following simple relations are noted.

$$
\begin{aligned}
& \phi_{-\alpha, P}^{*}(n)=\frac{\phi_{\alpha, P}(n)}{n^{\alpha}}, \\
& \sigma_{-\alpha, P}^{*}(n)=\frac{\sigma_{\alpha, P}(n)}{n^{\alpha}} .
\end{aligned}
$$

Corresponding to the case $P=1$, we place $\phi_{\alpha, 1}(n)=\phi_{\alpha}(n), \phi_{\alpha, 1}^{*}=\phi_{\alpha}^{*}(n)$, and corresponding to the case $P=Z$, we write $\sigma_{\alpha, Z}(n)=\sigma_{\alpha}(n)=\sigma_{\alpha, Z}^{*}(n)$.

The following result is a generalization of [3, Theorem $8, \alpha=1]$ and can be proved similarly.

THEOREM 2.3.

$$
\phi_{\alpha, P}(n)=\sum_{d \delta=n} \phi_{\alpha}(d) \rho_{P}(\delta) .
$$

We also note, by inversion of (2.7), the following generalization of (2.5).

$$
\sum_{a \delta=n} \rho_{Q}(d) \phi_{\alpha, P}(\delta)=n^{\alpha} .
$$

3. The zeta-functions of a $P$-set.

REMARK 3.1. In the definitions and general results of this section, $s$ is assumed to be limited to values for which all occuring series converge absolutely.

First we define for real $s$,

$$
\zeta_{P}(s)=\sum_{n=1}^{\infty} \frac{\rho_{P}(n)}{n^{s}}=\sum_{\substack{n=1 \\ n \in P}}^{\infty} \frac{1}{n^{s}} .
$$

The function $\zeta_{P}(s)$ will be called the zeta-function of the direct factor set $P$. Note that $\zeta_{z}(s)=\zeta(s), \zeta_{1}(s)=1$. We define the reciprocal zetafunction of $P$ by

$$
\tilde{\zeta}_{P}(s)=\sum_{n=1}^{\infty} \frac{\mu_{P}(n)}{n^{s}} ;
$$

the function $\zeta_{Q}(s)$ will be designated the conjugate zeta-function of $P$. 
By (1.1) it follows that $\tilde{\zeta}(s) \equiv \tilde{\zeta}_{1}(s)=1 / \zeta(s)$. We mention that Diricelet series of the form (3.1), (3.2) were discussed by Wintner [10, Chapter II] in case $P$ is a semigroup generated by a set of primes.

First we prove two relations analogous to (2.4).

LEMMA 3.1.

$$
\sum_{d S=n} \rho_{P}(d) \rho_{Q}(\delta)=1
$$

Proof. This is an immediate consequence of property (ii) of the conjugate pair $P, Q$.

LEMMA 3.2.

$$
\sum_{a S=n} \mu_{P}(d) \mu_{Q}(\delta)=\mu(n)
$$

Proof. By the definition of $\mu_{P}(n)$, we have, with the left member of (3.4) denoted by $S(n)$,

$$
\begin{aligned}
& S(n)=\sum_{a S=n} \sum_{\substack{D D^{\prime}=l \\
D^{\prime} \in P}} \mu(D) \sum_{\substack{E E^{\prime}=0 \\
E^{\prime} \in Q}} \mu(E)=\sum_{\substack{D D^{\prime} \\
D, E^{\prime} \in E^{\prime}=n \\
\nu^{\prime} \in P^{\prime} \in Q}} \mu(D) \mu(E) \\
& =\sum_{D E^{\prime} \mid n} \mu(D) \mu(E) \sum_{\substack{D^{\prime} \in E^{\prime}=n / D E \\
D^{\prime} \in E^{\prime} \in Q}} 1 .
\end{aligned}
$$

By property (ii), it follows then that

$$
S(n)=\sum_{D E \mid n} \mu(D) \mu(E)=\sum_{D \mid E} \mu(D) \sum_{E \mid(n / D)} \mu(E),
$$

and (3.4) results by the fundamental property of $\mu(n),((2.4)$ with $P=1$, $Q=Z)$.

The following relations are basic.

TheOREM 3.1 .

$$
\begin{aligned}
& \zeta_{P}(s) \zeta_{Q}(s)=\zeta(s), \\
& \tilde{\zeta}_{P}(s) \tilde{\zeta}_{Q}(s)=\zeta^{-1}(s), \\
& \zeta_{P}(s) \tilde{\zeta}_{Q}(s)=1 .
\end{aligned}
$$

Proof. By the nature of the Dirichlet product, (3.5), (3.6), and (3.7) follow, respectively, from (3.3), (3.4), and (2.4).

By Theorem 3.1 one obtains the following generalization of (1.1):

Corollary 3.1,

$$
\tilde{\zeta}_{P}(s)=\frac{\zeta_{P}(s)}{\zeta(s)}=\frac{1}{\zeta_{Q}(s)}
$$


The equality of the first two expressions in (3.8) is equivalent to the fact $[3,(4.6)]$,

$$
\sum_{a \mid n} \mu_{P}(d)=\rho_{P}(n) .
$$

The following identities can be verified by Dirichlet multiplication, in connection with (3.8), (2.13), and (2.11a).

THEOREM 3.2.

$$
\begin{aligned}
& \sum_{n=1}^{\infty} \frac{\phi_{\alpha, P}(n)}{n^{s}}=\frac{\zeta(s-\alpha)}{\zeta_{Q}(s)}=\frac{\zeta(s-\alpha) \zeta_{P}(s)}{\zeta(s)} ; \\
& \sum_{n=1} \frac{\phi_{\alpha, P}^{*}(n)}{n^{s}}=\frac{\zeta(s)}{\zeta_{Q}(s-\alpha)}=\frac{\zeta(s) \zeta_{P}(s-\alpha)}{\zeta(s-\alpha)} .
\end{aligned}
$$

THEOREM 3.3.

$$
\begin{aligned}
& \sum_{n=1}^{\infty} \frac{\sigma_{\alpha, P}(n)}{n^{s}}=\zeta(s-\alpha) \zeta_{P}(s) ; \\
& \sum_{n=1}^{\infty} \frac{\sigma_{\alpha, P}^{*}(n)}{n^{s}}=\zeta(s) \zeta_{P}(s-\alpha) .
\end{aligned}
$$

Note that in case $P=Z$, both (3.12) and (3.13) reduce to [7, Theorem 291|.

It is also noted, on the basis of (3.12) and (3.8), that

COROLLARY 3.3 .

$$
\zeta_{Q}(s) \sum_{n=1}^{\infty} \frac{\sigma_{\alpha, P}(n)}{n^{s}}=\sum_{n=1}^{\infty} \frac{\sigma_{\alpha}(n)}{n^{s}} .
$$

Multiplying (3.14) by $\tilde{\zeta}_{P}(s)$ and comparing coefficients, one obtains the arithmetical relation.

COROLLARY 3.4 .

$$
\sigma_{\alpha, P}(n)=\sum_{d \delta=n} \sigma_{\alpha}(d) \mu_{P}(\delta) .
$$

This analogue of (2.12) can also be proved arithmetically on the basis of (3.9) and the definition of $\sigma_{\alpha, P}(n)$.

In the remainder of this section, we list for later reference, explicit evaluations of $\zeta_{P}(s)$ for various sets $P$. Let $k$ and $r$ denote fixed positive integers and $p$ a fixed prime. We define direct factor sets $P=A_{k}, B_{k}$, $C_{p}, D_{r}, E_{r}$ as follows: $A_{k}$ (the set of $k$ th powers), $B_{k}$ (the set of $k$-free integers), $C_{p}$ (the non-negative powers of $p$ ), $D_{r}$ (the divisors of $r$ ), $\mathrm{E}_{r}$ (the complete divisors of $r$ ). A divisor $d$ of $r$ is said to be complete if $(d, r / d)=1$.

We have the following representations. 


$$
\begin{array}{ll}
\zeta_{A_{k}}(s)=\zeta(k s) & (k s>1), \\
\zeta_{B_{k}}(s)=\frac{\zeta(s)}{\zeta(k s)} & (s>1), \\
\zeta_{c_{p}}(s)=\frac{p^{s}}{p^{s}-1} & (s>0), \\
\zeta_{D_{r}}(s)=\frac{\sigma_{s}(r)}{r^{s}}=\sigma_{-s}(r), & \\
\zeta_{E_{r}}(s)=\frac{\sigma_{s}^{\prime}(r)}{r^{s}}=\sigma_{-s}^{\prime}(r), &
\end{array}
$$

where $\sigma_{s}^{\prime}(r)$ denotes the sum of the $s$ th powers of the complete divisors of $r$. For a proof of (3.17) we refer to [7, Theorem 303]; (3.18) results on summing a geometric series.

We mention the following special cases of (3.10) and (3.12), which result on the basis of (3.16) and (3.17), respectively.

$$
\begin{array}{ll}
\sum_{n=1}^{\infty} \frac{\phi_{\alpha, A_{k}}(n)}{n^{s}}=\frac{\zeta(s-\alpha) \zeta(k s)}{\zeta(s)} & (s>\alpha, s>1), \\
\sum_{n=1}^{\infty} \frac{\sigma_{\alpha, B_{k}}(n)}{n^{s}}=\frac{\zeta(s-\alpha) \zeta(s)}{\zeta(k s)} & (s>\alpha, s>1) .
\end{array}
$$

4. Mean values of totient and divisor functions. In this section we prove, along classical lines, some simple estimates for the functions introduced in $\S 2$. We require no more than the following elementary facts :

$$
\begin{aligned}
& \sum_{n \leq x} \frac{1}{n^{\alpha}}=\left\{\begin{array}{l}
O(1) \\
O(\log x) \\
O\left(x^{1-\alpha}\right)
\end{array}\right. \\
& \sum_{n \leq x} n^{\alpha}=\frac{x^{\alpha+1}}{\alpha+1}+\left\{\begin{array}{l}
O\left(x^{\alpha}\right) \\
O(1)
\end{array}\right. \\
& \sum_{n>x} \frac{1}{n^{\alpha}}=O\left(\frac{1}{x^{\alpha-1}}\right),
\end{aligned}
$$$$
\text { if } \alpha>1 \text {, }
$$$$
\text { if } \alpha=1 \text {, }
$$$$
\text { if } \alpha<1 \text {; }
$$$$
\text { if } \alpha \geqq 0 \text {, }
$$$$
\text { if }-1<\alpha<0 \text {; }
$$$$
\alpha>1 \text {. }
$$

LEMMA 4.1. For $P$ an arbitrary direct factor set, $\mu_{P}(n)$ is bounded; in fact, for each $n>0, \mu_{P}(n)=1,-1$, or 0 .

Proof. In view of the factorability [3, Theorem 1] of $\mu_{P}(n)$, it suffices to prove the lemma in case $n=p^{h}, p$ prime, $h>0$. We have then by (2.2),

$$
\mu_{P}\left(p^{h}\right)=\rho_{P}\left(p^{h}\right)-\rho_{P}\left(p^{h-1}\right)
$$


so that

$$
\mu_{P}\left(p^{h}\right)=\left\{\begin{array}{r}
1 \\
-1 \\
0
\end{array}\right.
$$$$
\left(p^{h} \in P, p^{h-1} \notin P\right)
$$$$
\left(p_{h} \notin P, p^{h-1} \in P\right)
$$$$
\text { (otherwise). }
$$

The lemma is proved.

As a consequence of Lemma 4.1, one obtains

COROLlaRY 4.1. The series (3.2) is absolutely convergent for $s>1$.

In the following, $x$ will be assumed $>1$.

ThEOREM 4.1. For all $\alpha>0$

$$
\begin{aligned}
& \sum_{n \leqq x} \phi_{\alpha, P}(n)=\left(\frac{x^{\alpha+1}}{\alpha+1}\right) \frac{1}{\zeta_{Q}(\alpha+1)}+O\left(e_{\alpha}(x)\right), \\
& \sum_{u \leqq x} \sigma_{\alpha, P}(n)=\left(\frac{x^{\alpha+1}}{\alpha+1}\right) \zeta_{P}(\alpha+1)+O\left(e_{\alpha}(x)\right),
\end{aligned}
$$

where

$$
e_{\alpha}(x)=\left\{\begin{array}{l}
x^{\alpha} \\
x \log x \\
x
\end{array}\right.
$$

Proof. We prove (4.5). By (2.7)

$$
\begin{aligned}
\Phi_{\alpha, P}(x) & \equiv \sum_{n \leqq x} \phi_{\alpha, P}(n)=\sum_{n \leqq x} \sum_{\substack{\delta ! n \\
(d \delta=n)}} \delta^{\alpha} \mu_{P}\left(\frac{n}{\delta}\right) \\
& =\sum_{d \delta \leqq x} \delta^{\alpha} \mu_{P}(d)=\sum_{d \leqq x} \mu_{P}(d) \sum_{\delta \leqq x / d} \delta^{\alpha} .
\end{aligned}
$$

Hence by (4.2) and Lemma 4.1,

$$
\begin{aligned}
\Phi_{\alpha, P}(x) & =\sum_{d \leqq x} \mu_{P}(d)\left\{\frac{(x / d)^{\alpha+1}}{\alpha+1}+O\left(\left(\frac{x}{d}\right)^{\alpha}\right)\right\} \\
& =\frac{x^{\alpha+1}}{\alpha+1} \sum_{d \leqq x} \frac{\mu_{P}(d)}{d^{\alpha+1}}+O\left(x^{\alpha} \sum_{d \leqq x} \frac{1}{d^{\alpha}}\right) .
\end{aligned}
$$

By (4.1) and Corollary 4.1, one may write then

$$
\Phi_{\alpha, P}(x)=\frac{x^{\alpha+1}}{\alpha+1}\left\{\tilde{\zeta}_{P}(\alpha+1)-\sum_{\alpha>x} \frac{\mu_{P}(d)}{d^{\alpha+1}}\right\}+\left(e_{\alpha}(x)\right) .
$$

But by Lemma 4.1 and (4.3), it follows that

$$
\sum_{d>x} \frac{\mu_{P}(d)}{d^{\alpha+1}}=O\left(\sum_{a>x} \frac{1}{d^{\alpha+1}}\right)=O\left(\frac{1}{x^{\alpha}}\right)
$$


for all $\alpha>0$. By (4.8), (4.9), and (3.8) the proof of (4.5) is complete. The proof of (4.6) is similar and the details will be omitted; likewise for the following result.

\section{Theorem 4.2. For all $\alpha>0$}

$$
\begin{aligned}
& \sum_{n \leqq x} \phi_{-\alpha, P}^{*}(n)=\frac{x}{\zeta_{Q}(\alpha+1)}+O\left(e_{\alpha}^{*}(x)\right), \\
& \sum_{n \leqq x} \sigma_{-\alpha, P}^{*}(n)=x \zeta_{P}(\alpha+1)+O\left(e_{\alpha}^{*}(x)\right),
\end{aligned}
$$

were $e_{\alpha}^{*}(x)=x^{-\alpha} e_{\alpha}(x)$ and $e_{\alpha}(x)$ is defined as in Theorem 4.1.

5. Asymptotic density of vector sets. We shall refer to the greatest common divisor $\left(a_{i}\right)$ of the components of a $Z_{t}$-vector $\left\{a_{i}\right\}$ as the index factor of the vector. Let $S$ be a set of positive integers and let $N_{t}(x, S)$ denote the number of $Z_{t}$-vectors with components $a_{i} \leqq x(i=1, \cdots, t)$ and with index factor in $S$. Then place

$$
\delta_{t}(S)=\lim _{x \rightarrow \infty} \frac{N_{t}(x, S)}{x^{6}},
$$

(if this limit exists) and call $\delta_{t}(S)$ the asymptotic density of the set of $Z_{t}$-vectors with index factor in $S$. We now prove the principal result of this section.

THEOREM 5.1. If $t$ is an integer $\geqq 2$, then

$$
N_{\iota}(x, P)=\frac{x^{b}}{\zeta_{Q}(t)}+ \begin{cases}O(x \log x) & \text { if } t=2, \\ O\left(x^{t-1}\right) & \text { if } t>2 .\end{cases}
$$

Proof. For positive integral $r, x \geqq 1$, place

$$
\Phi_{r, P}(x)=\sum_{n \leqq x} J_{r, P}(n)=\sum_{n \leqq x} \phi_{r, P}(n), \quad \Phi_{o, P}(x)=1 .
$$

Let $j$ be a fixed integer, $1 \leqq j \leqq t$, and let $i_{1}, \cdots, i_{j}$ be a set of distinct integers satisfying $1 \leqq i<\cdots<i_{j} \leqq t$. Consider all $Z_{t}$-vectors such that the components in the positions $i_{1}, \cdots, i_{j}$ have the same value $n$, the components in the remaining positions are $\leqq n$, and the index factor is in $P$. Denote by $S_{j}$ the set of all such vectors, including repetitions, obtained by letting $n$ range over the set, $1 \leqq n \leqq x$, and by choosing the set, $i_{1}, \cdots, i_{j}$, in every possible way. Then if $N\left(S_{j}\right)$ denotes the number of elements in $S_{\jmath}$, it follows that

$$
N\left(S_{j}\right)=\left(\begin{array}{l}
t \\
j
\end{array}\right) \Phi_{t-j, P}(x) .
$$


Consider now a fixed $Z_{t}$-vector, $\beta_{k} \in S_{k}, 1 \leqq k \leqq t$, with exactly $k$ of its components equal to $n$ and the remaining components $<n$. Then $\beta_{k}$ appears $\left(\begin{array}{l}k \\ j\end{array}\right)$ times in $S_{j}$, it being understood that $\left(\begin{array}{l}k \\ j\end{array}\right)=0$ if $j>k$. In view of the fact,

$$
\sum_{j=1}^{\iota}(-1)^{j+1}\left(\begin{array}{l}
k \\
j
\end{array}\right)=1,
$$

it follows that $\beta_{k}$ is contained exactly once in the set

$$
\sum_{j=1}^{t}(-1)^{j+1} S_{j} .
$$

Consequently

$$
N_{t}(x, P)=\sum_{j=1}^{t}(-1)^{j+1} N\left(S_{j}\right)
$$

hence by $(5.2)$,

$$
N_{\iota}(x, P)=\sum_{j=1}^{\iota}(-1)^{j+1}\left(\begin{array}{c}
t \\
j
\end{array}\right) \Phi_{t-j, P}(x) .
$$

The theorem follows by (4.5) on taking limits.

As a corollary of Theorem 5.1 one obtains by (3.8),

Corollary 5.1 (cf. [2, p. 8]). If $t \geqq 2$, then $\delta_{t}(P)$ exists and is given by

$$
\delta_{t}(P)=\frac{1}{\zeta_{Q}(t)}=\frac{\zeta_{P}(t)}{\zeta(t)} .
$$

As in $\S 3$ let $r$ and $k$ denote positive integers and $p$ a positive prime. On the basis of the evaluations (3.16)-(3.20), we obtain the following special cases of Corollary 5.1.

Corollary 5.2. The asymptotic density of the $Z_{t}$-vectors, $t \geqq 2$, (i) with index factor a kth power is

$$
\delta_{t}\left(A_{k}\right)=\frac{\zeta(k t)}{\zeta(t)}
$$

(ii) with k-free index factor is

$$
\delta_{t}\left(B_{k}\right)=\frac{1}{\zeta(k t)}
$$

(iii) with index factor a non-negative power of $p$ is

$$
\delta_{t}\left(C_{p}\right)=\left(\frac{p^{t}}{p^{t}-1}\right) \frac{1}{\zeta(t)}
$$


(iv) with index factor a divisor of $r$ is

$$
\delta_{t}\left(D_{r}\right)=\frac{\sigma_{t}(r)}{r^{t} \zeta(t)}
$$

(v) with index factor a complete divisor of $r$ is

$$
\delta_{t}\left(E_{r}\right)=\frac{\sigma_{t}^{\prime}(r)}{r^{t} \zeta(t)}=\frac{\sigma_{-t}^{\prime}(r)}{\zeta(t)} .
$$

The results contained in (5.4) and (5.5) are due originally to Gegenbauer [5]. In case $k=1$, (5.5) becomes $\delta_{t}\left(B_{1}\right)=1 / \zeta(t), t \geqq 2[9$, p. 156]. Further specialization of (5.5) to the case $k=1, t=2$ yields the classical result [7, Theorem 332] asserting that the probability that a pair of integers be relatively prime is $6 / \pi^{2}$. By (5.4), with $k=2, t=2$, it follows that the density of the integral pairs whose greatest common divisor is a perfect square is $\pi^{2} / 15$. The case $p=2, t=2$ in (5.6) shows that the density of the integral pairs with greatest common divisor a power of 2 is $8 / \pi^{2}$. By (5.7) with $r=8, t=2$, it follows that the density of the pairs of integers whose greatest common divisor is a factor of 8 is $255 / 32 \pi^{2}$.

Corollary 5.3. If $t \geqq 2$ and $r$ is a positive integer, then the asymptotic density of the $Z_{t}$-vectors with index factor $r$ is

$$
\delta_{t}(r)=\frac{1}{r^{t} \zeta(t)}
$$

Sketch of proof. The corollary is true in case $r=1$, as noted above on the basis of (5.5), or alternatively by (5.7) with $r=1$. The proof can be completed for arbitrary $r$ by induction on the number of distinct prime factors of $r$ and application of (5.8). The details are omitted.

The preceding corollary is due to Kronecker in case $t=2[8, \mathrm{p} .311]$. It was proved in the general case by Cesàro [1, p. 293]; a further generalization was given by G. Daniloff $[4$, p. 587].

6. Generalization of the second Möbius inversion formula. In case $P=1, Q=Z$, the following inversion relation reduces to a familiar analogue [7, Theorem 268] of the Möbius inversion formula.

THEOREM 6.1. Let $x$ denote a positive real variable; then

$$
f(x)=\sum_{n \leqq x} \rho_{Q}(n) g\left(\frac{x}{n}\right) \rightleftarrows g(x)=\sum_{n \leqq x} \mu_{P}(n) f\left(\frac{x}{n}\right) .
$$

Proof. Let $g(x)$ be defined as on the right of (6.1). Then 


$$
\begin{aligned}
\sum_{n \leqq x} \rho_{Q}(n) g\left(\frac{x}{n}\right) & =\sum_{n \leqq x} \rho_{Q}(n) \sum_{\substack{l \leq x / n \\
(l=n d)}} \mu_{P}(d) f\left(\frac{x / n}{d}\right) \\
& =\sum_{l \leqq x} f\left(\frac{x}{l}\right) \sum_{l=a n} \mu_{P}(d) \rho_{Q}(n)=f(x),
\end{aligned}
$$

on the basis of (2.4). The converse is proved similarly.

We define $[x]_{P}$ to be the number of positive integers $\leqq x$ belonging to $P$. It is evident, by property (ii) of the conjugate pair $P, Q$, that

$$
[x]=[x]_{Z}=\sum_{\substack{n \leq x \\ n \in Q}}\left[\frac{x}{n}\right]_{P}=\sum_{n \leq x}\left[\frac{x}{n}\right]_{P} \rho_{Q}(n) .
$$

Applying the above inversion theorem to (6.), one obtains

THEOREM 6.2.

$$
[x]_{P}=\sum_{n \leqq x} \mu_{P}(n)\left[\frac{x}{n}\right] .
$$

We deduce two special cases of (6.3). Let $A_{k}, B_{k}$ be the $P$-sets defined in $\S 3$ and place (as in $I), \lambda_{k}(n)=\mu_{A_{k}}(n), \mu_{k}(n)=\mu_{B_{k}}(n)$. Putting $[x]_{k}=[x]_{B_{k}}$ and nothing that $[\sqrt{k} \bar{x}]=[x]_{A_{k}}$, one obtains

COROLLARY 6.1 .

$$
\begin{gathered}
{[x]_{k}=\sum_{n \leqq x} \mu_{k}(n)\left[\frac{x}{d^{k}}\right]=\sum_{d^{k} \leqq x} \mu(d)\left[\frac{x}{d^{k}}\right],} \\
{[\sqrt[k]{x}]=\sum_{n \leqq x} \lambda_{k}(n)\left[\frac{x}{n}\right] .}
\end{gathered}
$$

These formulas are classical [6], [9, p. 35]. Note that (6.4) and (6.5) reduce to (1.3) in the cases $k=1$ and $k=0$, respectively.

It can be shown easily, on the basis of (6.4), that $\delta_{1}\left(B_{k}\right)=1 / \zeta(k)$, $k>1$ (cf. [7, Theorem 333] in case $k=2$ ). In words, this states that the asymptotic density of the $k$-free integers $(k \geqq 2)$ is $1 / \zeta(k)$; in conjunction with (5.5) it therefore follows that

COROLlaRY 6.2. If $k t \geqq 2$, then the asymptotic density of the $Z_{t^{-}}$ vectors with $k$-free index factor is $1 / \zeta(k t)$.

Finally, we consider the function $\phi_{P}(x, n)$ defined to be the number of positive integers $a \leqq x$ such that $(a, n) \in P$. In case $P=1, \phi_{P}(x, n)$ becomes Legendre's function $\phi(x, n)$. To deal with $\phi_{P}(x, n)$ we have the following extension of [3, Theorem 4] which can be proved in much the same way.

LEMMA 6.1. Let $d$ range over the divisors of $n, d \in Q$, and for 
each such $d$, let $y$ range over the positive integers $a \leqq x / d$ such that $(a, n / d) \in P$. Then the set $d y$ consists of the positive integers $\leqq x$

An immediate consequence of this lemma is

THEOREM 6.3.

$$
\sum_{d \mid n} \phi_{P}\left(\frac{x}{d}, \frac{n}{d}\right) \rho_{Q}(d)=[x]
$$

THEOREM 6.4 .

$$
\phi_{P}(x, n)=\sum_{d \mid n} \mu_{P}(d)\left[\frac{x}{d}\right]
$$

Theorem 6.4 can be deduced from (6.6) by a direct application of the following easily proved extension of (2.3).

THEOREM 6.5. If $f(x, n)$ and $g(x, n)$ are functions of the real variable $x$ and the positive integral variable $n$, then

$$
g(x, n)=\sum_{d \mid n} \rho_{Q}(d) f\left(\frac{x}{d}, \frac{n}{d}\right) \rightleftarrows f(x, n)=\sum_{d \mid n} \mu_{P}(d) g\left(\frac{x}{d}, \frac{n}{d}\right) .
$$

The proof is omitted.

\section{BIBLIOGRAPHY}

1. Ernest Cesàro, Sur le plus grand commun diviseur de plusieurs nombres, Annali di Matematica, 13 (1885), II, 291-294.

2. — Sur l'étude des événements arithmétiques, Mémoires de l'académie royale des sciences de Belgique, 47 (1886), 13 pages.

3. Eckford Cohen, $A$ class of residue systems $(\bmod r)$ and related arithmotical functions,

I. A generalization of Möbius inversion, Pacific J. Math., 9 (1959), 13-23.

4. Georges Daniloff, Contribution à la théorie des fonctions arilhmétiques, Recueil de l'Académie des Sciences Bulgare, 35 (1941), 479-590. (Bulgarian, French summary).

5. Leopold Gegenbauer, Einige asymptotische Gesetze der Zahlentheorie, Sitzungsberichte der Akademie der Wissenschaften (Vienna), 92 (1885), 1290-1306.

6. ——Über die Anzahl der Primzahlen, Sitzungsberichte der Akademie der Wissenschaften (Vienna), 95 (1887), 94-96.

7. G. H. Hardy and E. M. Wright, Introduction to the Theory of Numbers (3rd edition), Oxford, 1954.

8. Leopold Kronecker, Vorlesungen über Zahlentheorie, Leipzig, 1901.

9. I. M. Vinogradov, Elements of Number Theory, New York, 1954, (English translation).

10. Aurel Wintner, The Theory of Measure in Arithmetical Semigroups, Baltimore, 1944.

The University of Tennessee 



\section{PACIFIC JOURNAL OF MATHEMATICS}

\section{EDITORS}

\section{David Gilbarg}

Stanford University Stanford, California

\section{R. A. Beaumont}

University of Washington

Seattle 5, Washington

\section{A. L. Whiteman}

University of Southern California Los Angeles 7, California

L. J. Paige

University of California

Los Angeles 24, California

\section{ASSOCIATE EDITORS}

E. F. BECKENBACH
C. E. BURGESS
E. HEWITT
A. HORN

A. HORN

\author{
V. GANAPATHY IYER \\ R. D. JAMES \\ M. S. KNEBELMAN \\ L. NACHBIN
}

I. NIVEN

T. G. OSTROM

H. L. ROYDEN

M. M. SCHIFFER
E. G. STRAUS

G. SZEKERES

F. WOLF

K. YOSIDA

\section{SUPPORTING INSTITUTIONS}

\author{
UNIVERSITY OF BRITISH COLUMBIA \\ CALIFORNIA INSTITUTE OF TECHNOLOGY \\ UNIVERSITY OF CALIFORNIA \\ MONTANA STATE UNIVERSITY \\ UNIVERSITY OF NEVADA \\ OREGON STATE COLLEGE \\ UNIVERSITY OF OREGON \\ OSAKA UNIVERSITY \\ UNIVERSITY OF SOUTHERN CALIFORNIA
}

\author{
STANFORD UNIVERSITY \\ UNIVERSITY OF TOKYO \\ UNIVERSITY OF UTAH \\ WASHINGTON STATE COLLEGE \\ UNIVERSITY OF WASHINGTON \\ * * * * \\ AMERICAN MATHEMATICAL SOCIETY \\ CALIFORNIA RESEARCH CORPORATION \\ HUGHES AIRCRAFT COMPANY \\ SPACE TECHNOLOGY LABORATORIES
}

Mathematical papers intended for publication in the Pacific Journal of Mathematics should be typewritten (double spaced), and the author should keep a complete copy. Manuscripts may be sent to any one of the four editors. All other communications to the editors should be addressed to the managing editor, L. J. Paige at the University of California, Los Angeles 24, California.

50 reprints per author of each article are furnished free of charge; additional copies may be obtained at cost in multiples of 50 .

The Pacific Journal of Mathematics is published quarterly, in March, June, September, and December. The price per volume (4 numbers) is $\$ 12.00$; single issues, $\$ 3.50$. Back numbers are available. Special price to individual faculty members of supporting institutions and to individual members of the American Mathematical Society: $\$ 4.00$ per volume; single issues, $\$ 1.25$.

Subscriptions, orders for back numbers, and changes of address should be sent to Pacific Journal of Mathematics, 2120 Oxford Street, Berkeley 4, California.

Printed at Kokusai Bunken Insatsusha (International Academic Printing Co., Ltd.), No. 6, 2-chome, Fujimi-cho, Chiyoda-ku, Tokyo, Japan.

PUBLISHED BY PACIFIC IOURNAL OF MATHEMATICS, A NON-PROFIT CORPORATION

The Supporting Institutions listed above contribute to the cost of publication of this Journal, but they are not owners or publishers and have no responsibility for its content or policies. 


\section{Pacific Journal of Mathematics}

\section{Vol. 9, No. $3 \quad$ July, 1959}

Errett Albert Bishop, A minimal boundary for function algebras . . . . . . . . . . . . 629

John W. Brace, The topology of almost uniform convergence . . . . . . . . . . . . 643

Cecil Edmund Burgess, Chainable continua and indecomposability .......... 653

L. Carlitz, Multiplication formulas for products of Bernoulli and Euler

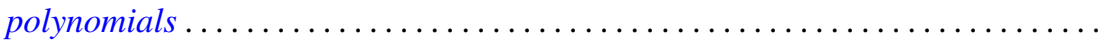

Eckford Cohen, A class of residue systems (mod $r$ ) and related arithmetical

functions. II. Higher dimensional analogues ....................

Shaul Foguel, Boolean algebras of projections of finite multiplicity . . . . . . . . . .

Richard Robinson Goldberg, Averages of Fourier coefficients .................

Seymour Goldberg, Ranges and inverses of perturbed linear operators .

Philip Hartman, On functions representable as a difference of convex functions ....

Milton Vernon Johns, Jr. and Ronald Pyke, On conditional expectation and

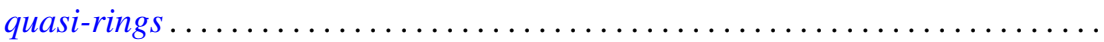

Robert Jacob Koch, Arcs in partially ordered spaces ....................

Gregers Louis Krabbe, A space of multipliers of type $L^{p}(-\infty, \infty) \ldots \ldots \ldots \ldots$

John W. Lamperti and Patrick Colonel Suppes, Chains of infinite order and their

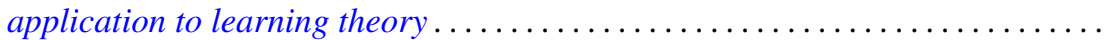

Edith Hirsch Luchins, On radicals and continuity of homomorphisms into Banach

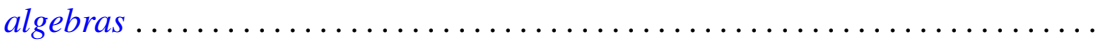

T. M. MacRobert, Multiplication formulae for the E-functions functions of their parameters.

Michael Bahir Maschler, Classes of minimal and representative domains and their kernel functions.

William Schumacher Massey, On the imbeddability of the real projective spaces in Euclidean space.

Thomas Wilson Mullikin, Semi-groups of class $\left(C_{0}\right)$ in $L_{p}$ determined by parabolic differential equations

Steven Orey, Recurrent Markov chains

Ernest Tilden Parker, On quadruply transitive groups ........ . .

Calvin R. Putnam, On Toeplitz matrices, absolute continuity, and unitary

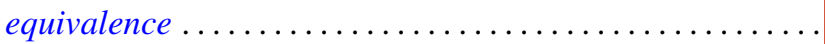

Helmut Heinrich Schaefer, On nonlinear positive operators.

Robert Seall and Marion Wetzel, Some connections between continued fractions and convex sets

Robert Steinberg, Variations on a theme of Chevalley

Olga Taussky and Hans Zassenhaus, On the similarity transformation between a

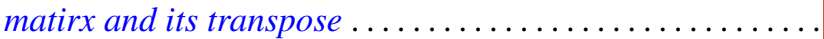

Emery Thomas, The suspension of the generalized Pontrjagin cohomology

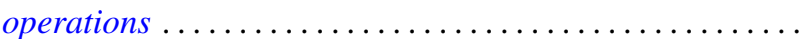

Joseph L. Ullman, On Tchebycheff polynomials ..................... 913

Richard Steven Varga, Orderings of the successive overrelaxation scheme ........ 925

Orlando Eugenio Villamayor, Sr., On weak dimension of algebras . 